\title{
An easy direct arylation of 5-pyrazolones
}

\author{
Hao Gong ${ }^{1}$, Yiwen Yang ${ }^{1,2}$, Zechao Wang ${ }^{1}$ and Chunxiang Kuang ${ }^{* 1,3}$
}

\section{Full Research Paper}

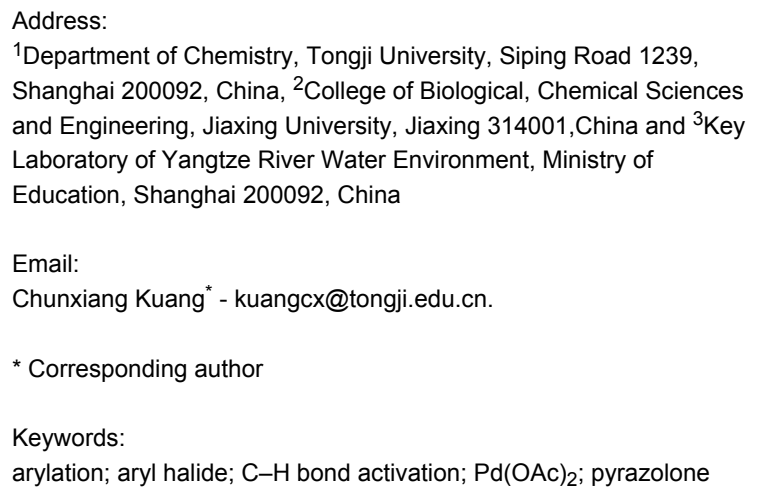

Beilstein J. Org. Chem. 2013, 9, 2033-2039.

doi:10.3762/bjoc. 9.240

Received: 14 July 2013

Accepted: 13 September 2013

Published: 08 October 2013

Associate Editor: M. Rueping

(C) 2013 Gong et al; licensee Beilstein-Institut. License and terms: see end of document.

\begin{abstract}
A mild, efficient and catalytic ligand-free method for the direct arylation of 5-pyrazolones by Pd-catalyzed C-H bond activation is reported. The process smoothly proceeds and yields are moderate to excellent.
\end{abstract}

\section{Introduction}

5-Pyrazolones are attracting considerable research interest because of their unique chemical properties and their structures that facilitate their application as biological and pharmaceutical intermediates and products [1-3]. Over the years, many of the biological activities of pyrazolones such as their antipyretic, analgesic [4,5], anti-inflammatory [6,7], antitumor [8,9], antiviral, antibacterial [10], and herbicidal [11] properties have been discovered and investigated. Pyrazolones are also potent inhibitors of telomerase, cyclooxygenase isoenzymes, platelet tromboxane synthesis, and prostanoid synthesis in humans $[12,13]$. Recently, pharmacologists have developed a novel class-II c-met inhibitor, whose structural unit is a pyrazolone ring [14]. The great medicinal significance and broad applications of pyrazolones prompted us to synthesize a new series of heterocyclic compounds containing the pyrazolone moiety.
The reaction of pyrazolones with arylboronic acids is an attractive approach for the synthesis of arylpyrazolone $[15,16]$. However, it often needs pre-formation of halo-pyrazolones. Transition metal-catalyzed direct arylation of (hetero)arenes has emerged over the past few years as a rapidly growing field of syntheses [17-26]. The direct arylation of pyrazolones by using aryl halides offers a cleaner and more efficient method of meeting such goals and rare examples of such transformations have been described [15].

In this paper, we report a convenient and catalytic ligand-free synthesis of a series of 4-aryl-5-pyrazolones 3 from 5-pyrazolones 1 and aryl halides 2 (Scheme 1). The direct arylation of 5-pyrazolones by $\mathrm{Pd}$-catalyzed $\mathrm{C}-\mathrm{H}$ bond activation was utilized. 


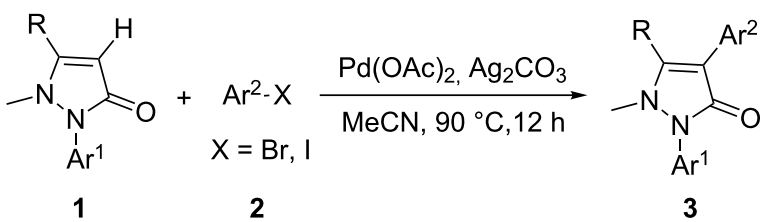

Scheme 1: Direct arylation of 5-pyrazolones.

\section{Results and Discussion}

We commenced this study by performing the direct arylation of phenazone (1a) in the presence of 2 equiv of iodobenzene (2a), $10 \mathrm{~mol} \%$ of $\mathrm{Pd}(\mathrm{OAc})_{2}$ as a catalyst in acetonitrile in a sealed tube. The results are shown in Table 1. Gratifyingly, a 45\% yield of the desired product $\mathbf{3 a}$ was achieved after stirring for $12 \mathrm{~h}$ at $90{ }^{\circ} \mathrm{C}$. Encouraged by this preliminary result, we continued to optimize reaction conditions to further improve the chemical yield.

When 1a reacted with $\mathbf{2} \mathbf{a}$ in the presence of $\mathrm{K}_{2} \mathrm{CO}_{3}$ as a base in acetonitrile $\left(90{ }^{\circ} \mathrm{C}, 12 \mathrm{~h}\right)$, the desired product 3a was generated in $43 \%$ yield (Table 1 , entry 2). Changing $\mathrm{K}_{2} \mathrm{CO}_{3}$ to $\mathrm{Cs}_{2} \mathrm{CO}_{3}$, $\mathrm{Na}_{2} \mathrm{CO}_{3}$ and DBU (1,8-diazabicyclo(5.4.0)undec-7-ene), decreased the yield to $35 \%, 27 \%$ and $0 \%$, respectively (Table 1 , entry 3-5). Changing $\mathrm{K}_{2} \mathrm{CO}_{3}$ to $\mathrm{K}_{3} \mathrm{PO}_{4}$, the yield was increased to $49 \%$ (Table 1, entry 6). When $\mathrm{Ph}_{3} \mathrm{P}$ as a catalytic ligand was added to the reaction, the yield decreased to $42 \%$ (Table 1 , entry 7). Reducing the dosage of $\mathrm{Pd}(\mathrm{OAc})_{2}$ to 0.05 equiv and 0.02 equiv, respectively, decreased the yield to $40 \%$ and $32 \%$ (Table 1, entries 8-9). Several solvents were examined under the conditions of entry 1 . When the solvent was changed to THF, DCE, dioxane, and benzene, the yields decreased to trace,

Table 1: Optimization of the synthesis of $3 a^{a}$

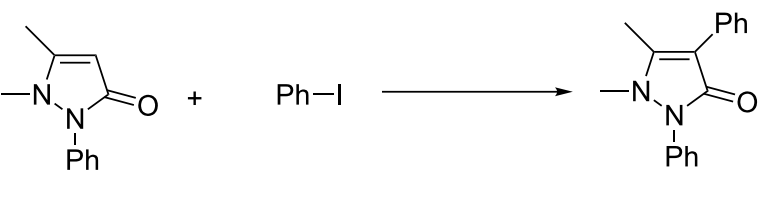

$1 \mathbf{a}$

$\mathbf{2 a}$

$3 a$

\begin{tabular}{|c|c|c|c|c|c|}
\hline entry & additive (2 equiv) & catalyst ( 0.1 equiv) & solvent & $T\left({ }^{\circ} \mathrm{C}\right)$ & yield of $3 a^{b}$ \\
\hline 1 & none & $\mathrm{Pd}(\mathrm{OAc})_{2}$ & $\mathrm{CH}_{3} \mathrm{CN}$ & 90 & 45 \\
\hline 2 & $\mathrm{~K}_{2} \mathrm{CO}_{3}$ & $\mathrm{Pd}(\mathrm{OAc})_{2}$ & $\mathrm{CH}_{3} \mathrm{CN}$ & 90 & 43 \\
\hline 3 & $\mathrm{Cs}_{2} \mathrm{CO}_{3}$ & $\mathrm{Pd}(\mathrm{OAc})_{2}$ & $\mathrm{CH}_{3} \mathrm{CN}$ & 90 & 35 \\
\hline 4 & $\mathrm{Na}_{2} \mathrm{CO}_{3}$ & $\mathrm{Pd}(\mathrm{OAc})_{2}$ & $\mathrm{CH}_{3} \mathrm{CN}$ & 90 & 27 \\
\hline 5 & $\mathrm{DBU}$ & $\mathrm{Pd}(\mathrm{OAc})_{2}$ & $\mathrm{CH}_{3} \mathrm{CN}$ & 90 & 0 \\
\hline 6 & $\mathrm{~K}_{3} \mathrm{PO}_{4}$ & $\mathrm{Pd}(\mathrm{OAc})_{2}$ & $\mathrm{CH}_{3} \mathrm{CN}$ & 90 & 49 \\
\hline 7 & $\mathrm{Ph}_{3} \mathrm{P}(0.25$ equiv $)$ & $\mathrm{Pd}(\mathrm{OAc})_{2}$ & $\mathrm{CH}_{3} \mathrm{CN}$ & 90 & 42 \\
\hline 8 & none & $\mathrm{Pd}(\mathrm{OAc})_{2}$ (0.05 equiv) & $\mathrm{CH}_{3} \mathrm{CN}$ & 90 & 40 \\
\hline 9 & none & $\mathrm{Pd}(\mathrm{OAc})_{2}$ (0.02 equiv) & $\mathrm{CH}_{3} \mathrm{CN}$ & 90 & 32 \\
\hline 10 & none & $\mathrm{Pd}(\mathrm{OAc})_{2}$ & THF & 90 & traces \\
\hline 11 & none & $\mathrm{Pd}(\mathrm{OAc})_{2}$ & DCE & 90 & 31 \\
\hline 12 & none & $\mathrm{Pd}(\mathrm{OAc})_{2}$ & dioxane & 90 & 0 \\
\hline 13 & none & $\mathrm{Pd}(\mathrm{OAc})_{2}$ & benzene & 90 & 22 \\
\hline 14 & none & $\mathrm{Pd}(\mathrm{OAc})_{2}$ & $\mathrm{CH}_{3} \mathrm{CN}$ & 25 & 0 \\
\hline 15 & none & $\mathrm{Pd}(\mathrm{OAc})_{2}$ & $\mathrm{CH}_{3} \mathrm{CN}$ & 60 & 31 \\
\hline 16 & none & $\mathrm{Pd}(\mathrm{OAc})_{2}$ & $\mathrm{CH}_{3} \mathrm{CN}$ & 120 & 35 \\
\hline 17 & $\mathrm{O}_{2}(1 \mathrm{~atm})$ & $\mathrm{Pd}(\mathrm{OAc})_{2}$ & $\mathrm{CH}_{3} \mathrm{CN}$ & 90 & 55 \\
\hline 18 & $\mathrm{~K}_{2} \mathrm{~S}_{2} \mathrm{O}_{8}$ & $\mathrm{Pd}(\mathrm{OAc})_{2}$ & $\mathrm{CH}_{3} \mathrm{CN}$ & 90 & 5 \\
\hline 19 & benzoquinone & $\mathrm{Pd}(\mathrm{OAc})_{2}$ & $\mathrm{CH}_{3} \mathrm{CN}$ & 90 & 0 \\
\hline 20 & $\mathrm{Cu}(\mathrm{OAc})_{2}$ & $\mathrm{Pd}(\mathrm{OAc})_{2}$ & $\mathrm{CH}_{3} \mathrm{CN}$ & 90 & 25 \\
\hline 21 & $\mathrm{Ag}_{2} \mathrm{CO}_{3}$ & $\mathrm{Pd}(\mathrm{OAc})_{2}$ & $\mathrm{CH}_{3} \mathrm{CN}$ & 90 & 80 \\
\hline 22 & none & $\mathrm{FeCl}_{3}$ (0.3 equiv) & $\mathrm{CH}_{3} \mathrm{CN}$ & 90 & 0 \\
\hline 23 & none & $\mathrm{Cu}(\mathrm{OAc})_{2}$ (0.2 equiv) & $\mathrm{CH}_{3} \mathrm{CN}$ & 90 & 0 \\
\hline 24 & none & none & $\mathrm{CH}_{3} \mathrm{CN}$ & 90 & 0 \\
\hline
\end{tabular}

aReaction conditions: 1.0 equiv of $1 \mathrm{a}$ and 2.0 equiv of $2 \mathrm{a}$ were stirred for $12 \mathrm{~h}$. ${ }^{\mathrm{b}}$ /solated yield. 
$31 \%, 0 \%$ and $22 \%$, respectively (Table 1 , entries $10-13$ ). Other reaction parameters such as temperature and oxidants were also screened. When the reaction temperatures were $25{ }^{\circ} \mathrm{C}, 60{ }^{\circ} \mathrm{C}$, and $120{ }^{\circ} \mathrm{C}$, the yields decreased to $0 \%, 31 \%$ and $35 \%$, respectively (Table 1, entries 14-16). When the reaction was under oxygen (1 atm) in a sealed tube and oxygen was used as an oxidant, product 3a was obtained in $55 \%$ yield (Table 1 , entry 17). Changing the oxidant to $\mathrm{K}_{2} \mathrm{~S}_{2} \mathrm{O}_{8}$, benzoquinone and $\mathrm{Cu}(\mathrm{OAc})_{2}$ decreased the yield to $5 \%, 0 \%$ and $25 \%$, respectively (Table 1, entries 18-20). When $\mathrm{Ag}_{2} \mathrm{CO}_{3}$ was added to the reaction, the yield increased to $80 \%$ (Table 1 , entry 21 ). Different catalysts were also examined. When $\mathrm{Cu}(\mathrm{OAc})_{2}$ or $\mathrm{FeCl}_{3}$ was used as a catalyst, or no catalyst was used in the reaction, product 3a was not obtained (Table 1, entries 22-24). Ultimately, the optimal reaction conditions were determined to be 0.1 equiv $\mathrm{Pd}(\mathrm{OAc})_{2}$ catalyst, 2.0 equiv $\mathrm{Ag}_{2} \mathrm{CO}_{3}$, acetonitrile,
$90{ }^{\circ} \mathrm{C}$, air atmosphere, 1:2 molar ratio of $1 \mathrm{a}$ to $2 \mathbf{a}$, and $12 \mathrm{~h}$ reaction time.

Under the optimized conditions (Table 1, entry 10), the scope of aryl halides was examined and the results are summarized in Table 2. The reactions of aryl halides 2 with phenyl moieties carrying either an electron-donating group such as methyl (2d and $\mathbf{2 i}$ ), ethyloxy (2e) or an electron-withdrawing substituent such as methoxycarbonyl (2c and $\mathbf{2 g}$ ), trifluoromethyl (2f) or formyl (2h) proceeded smoothly with moderate to good yields (Table 2, entries 3-10). When the phenyl moiety of the aryl halides 2 carried an electron-donating group, higher yields were obtained (Table 2, entries 4, 5, 9). On the other hand, an electron-withdrawing group on the phenyl moiety of the aryl halides (2c, $2 \mathbf{f}, 2 \mathrm{~g}$ and $\mathbf{2 h}$ ) provided 4-aryl-5-pyrazolones $\mathbf{3}$ in relatively low yields (Table 2, entries 3,6-8). Entries 1 and 2 show

Table 2: Synthesis of 4-aryl- 5-pyrazolones 3

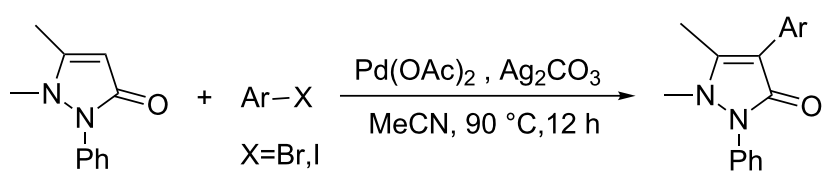

1a

2

3

entry yield of 3 (\%) $)^{a}$


Table 2: Synthesis of 4-aryl- 5-pyrazolones 3. (continued)

5

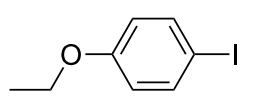

$2 e$

6
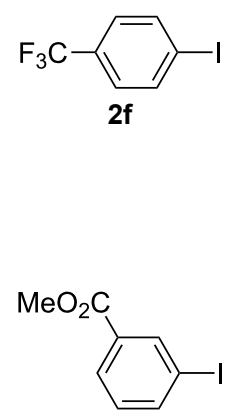

$2 \mathrm{~g}$

8

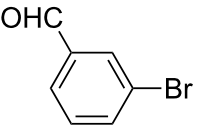

2h

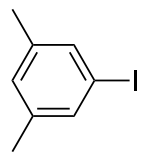

$2 \mathbf{i}$

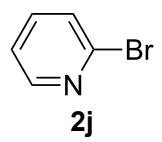<smiles>CCOc1ccc(-c2c(C)n(C)n(-c3ccccc3)c2=O)cc1</smiles>

3d<smiles>Cc1c(-c2ccc(C(F)(F)F)cc2)c(=O)n(-c2ccccc2)n1C</smiles>

$3 e$<smiles>CC(=O)c1cccc(-c2c(C)n(C)n(C)c2=O)c1</smiles>

$\mathrm{Ph}$<smiles>Cc1c(-c2cccc(C=O)c2)c(=O)n(-c2ccccc2)n1C</smiles>

$3 g$<smiles>Cc1cc(C)cc(-c2c(C)n(C)n(-c3ccccc3)c2=O)c1</smiles>

3h<smiles>Cc1c(-c2ccccn2)c(=O)n(-c2ccccc2)n1C</smiles>

84

asolated yield.

that the yield of products was lower when using aryl bromide than when using aryl iodide, and 2-bromopyridine also provided $3 \mathbf{i}$ in moderate yield (Table 2 , entry 10 ).

Next, we investigated the scope of 5-pyrazolone 1 substrates. Table 3 shows that in most cases, the desired pyrazolones $\mathbf{3}$ were generated smoothly in moderate to good yields. When the phenyl moiety of pyrazolones 1 carried an electron-donating substituent such as methoxy (1b) and methyl (1c), the reactions provided pyrazolones 3 in high yields (Table 3 , entries 1, 2). On the other hand, when pyrazolones 1 carried an electron-withdrawing substituent such as nitro (1f) and halogens (1g, 1i and 
Table 3: Synthesis of 4-phenyl-5-pyrazolones 3.

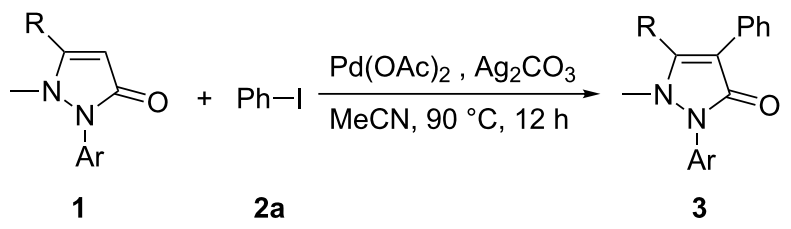

1

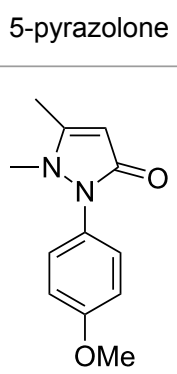

$1 \mathrm{~b}$<smiles>Cc1ccc(-n2c(=O)cc(C)n2C)cc1</smiles>

$1 \mathrm{c}$

$$
\mathrm{Ph}
$$

3<smiles>Cn1c(-c2ccccc2)cc(=O)n1-c1ccccc1</smiles>

4<smiles>CCCc1cc(=O)n(C)n1C</smiles>

$\mathrm{Ph}$

$$
1 e
$$<smiles>Cc1cc(=O)n(-c2ccc([N+](=O)[O-])cc2)n1C</smiles>

$1 f$

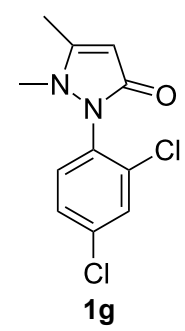

5

6 product<smiles>COc1ccc(-n2c(=O)c(-c3ccccc3)c(C)n2C)cc1</smiles>

3j<smiles>Cc1c(-c2ccccc2)c(=O)[nH]n1C</smiles><smiles>Brc1ccc(I)cc1</smiles>

83<smiles>Cn1c(-c2ccccc2)c(-c2ccccc2)c(=O)n1-c1ccccc1</smiles>

3I<smiles>CCCc1c(-c2ccccc2)c(=O)n(-c2ccccc2)n1C</smiles>

66<smiles>Cc1c(-c2ccccc2)c(=O)n(-c2ccc([N+](=O)[O-])cc2)n1C</smiles>

$3 n$

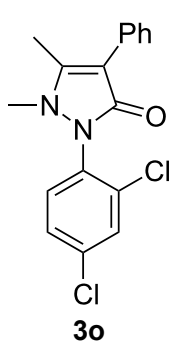

87 53

66


Table 3: Synthesis of 4-phenyl-5-pyrazolones 3. (continued)<smiles>Cn1c(-c2ccccc2)cc(=O)n1-c1ccc(Cl)cc1Cl</smiles>

1h

8<smiles>Cc1cc(=O)n(-c2ccc(F)cc2)n1C</smiles>

$1 \mathrm{i}$<smiles>CCCc1cc(=O)[nH]n1C</smiles>

9

10

11<smiles>CCN(C)c1ccc(F)cc1</smiles>

1j<smiles>Cc1cc(=O)n(-c2cccc(Cl)c2)n1C</smiles>

$1 \mathrm{k}$

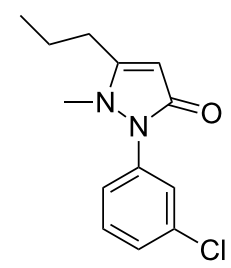<smiles>Cn1c(-c2ccccc2)c(-c2ccccc2)c(=O)n1-c1ccc(Cl)cc1Cl</smiles>

$3 p$<smiles>Cc1c(-c2ccccc2)c(=O)n(-c2ccc(F)cc2)n1C</smiles>

$3 q$<smiles>CCCc1c(-c2ccccc2)c(=O)n(-c2ccc(F)cc2)n1C</smiles>

$3 r$<smiles>Cc1c(-c2ccccc2)c(=O)n(-c2cccc(Cl)c2)n1C</smiles>

3s<smiles>CCCc1c(-c2ccccc2)c(=O)n(-c2cccc(Cl)c2)n1C</smiles>

$3 t$

alsolated yield.

$\mathbf{1 k}$ ) in the aromatic portion, relatively low yields were obtained (Table 3, entries 5, 6, 8, 10). Compared with 5-pyrazolones containing a butyl or a phenyl substituent on the 3-position of the heterocycle (1d and 1e), the methyl (1a) on the same position resulted in a higher yield (Table 3 , entries 3 and 4). The cause might be the steric hindrance of phenyl or butyl. The same trend could be seen from $\mathbf{1 g}$ to $\mathbf{1 l}$ ( $\mathrm{cf}$. 3o, $\mathbf{3 q}$ and $\mathbf{3 s}$ with 3p, 3r and 3t) (Table 3, entries 6-11).

\section{Conclusion}

In summary, we developed a mild, simple and efficient method for the direct arylation of 5-pyrazolones by Pd-catalyzed $\mathrm{C}-\mathrm{H}$ bond activation. This approach resulted in the construction of 4-aryl-5-pyrazolones, which are important heterocyclic compounds used in medicinal and biological research. The investigations on the reaction mechanism are still in progress. 


\section{Supporting Information}

\section{Supporting Information File 1}

Experimental details and characterization data for all compounds.

[http://www.beilstein-journals.org/bjoc/content/ supplementary/1860-5397-9-240-S1.pdf]

\section{Acknowledgements}

The present work was supported by the Natural Science Foundation of China (No. 21272174), the Key Projects of Shanghai in Biomedicine (No. 08431902700), and the Scientific Research Foundation of the State Education Ministry for Returned Overseas Chinese Scholars. We would also like to thank the Center for Instrumental Analysis, Tongji University, China.

\section{References}

1. Marinozzi, M.; Carotti, A.; Sansone, E.; Macchiarulo, A.; Rosatelli, E.; Sardella, R.; Natalini, B.; Rizzo, G.; Adorini, L.; Passeri, D.; De Franco, F.; Pruzanski, M.; Pellicciari, R. Bioorg. Med. Chem. 2012, 20, 3429-3445. doi:10.1016/j.bmc.2012.04.021

2. Dow, R. L.; Carpino, P. A.; Gautreau, D.; Hadcock, J. R.; Iredale, P. A.; Kelly-Sullivan, D.; Lizano, J. S.; O' Connor, R. E.; Schneider, S. R.; Scott, D. O.; Ward, K. M. ACS Med. Chem. Lett. 2012, 3, 397-401. doi:10.1021/ml3000325

3. Panda, N.; Karmakar, S.; Jena, A. K. Chem. Heterocycl. Compd. 2011, 46, 1500-1508. doi:10.1007/s10593-011-0699-y

4. Uramaru, N.; Shigematsu, H.; Toda, A.; Eyanagi, R.; Kitamura, S.; Ohta, S. J. Med. Chem. 2010, 53, 8727-8733. doi:10.1021/jm101208x

5. Gold, M.; McKeen, C.; Beaver, W. T. Am. J. Med. Sci. 1965, 250, 577-604. doi:10.1097/00000441-196511000-00011

6. Himly, M.; Jahn-Schmid, B.; Pittertschatscher, K.; Bohle, B.; Grubmayr, K.; Ferreira, F.; Ebner, H.; Ebner, C. J. Allergy Clin. Immunol. 2003, 111, 882-888. doi:10.1067/mai.2003.163

7. Marković, V.; Erić, S.; Stanojković, T.; Gligorijević, N.; Arandelović, S.; Todorović, N.; Trifunović, S.; Manojlović, N.; Jelić, R.; Joksović, M. D. Bioorg. Med. Chem. Lett. 2011, 21, 4416-4421. doi:10.1016/j.bmcl.2011.06.025

8. Braña, M. F.; Gradillas, A.; Ovalles, A. G.; López, B.; Acero, N.; Llinares, F.; Muñoz Mingarro, D. Bioorg. Med. Chem. 2006, 14, 9-16. doi:10.1016/j.bmc.2005.09.059

9. Tripathy, R.; Ghose, A.; Singh, J.; Bacon, E. R.; Angeles, T. S.; Yang, S. X.; Albom, M. S.; Aimone, L. D.; Herman, J. L.; Mallamo, J. P. Bioorg. Med. Chem. Lett. 2007, 17, 1793-1798. doi:10.1016/j.bmcl.2006.12.054

10. Sayed, G. H.; Shiba, S. A.; Radwan, A.; Mohamed, S. M.; Khalil, M. Chin. J. Chem. 1992, 10, 475-480. doi:10.1002/cjoc.19920100515

11. Vassilev, G. N.; Yonova, P. A.; Bohland, H.; Vassilev, N. G.; Yordanov, B. Dokl. Bulg. Akad. Nauk. 1997, 50, 59-62.

12. Costa, D.; Marques, A. P.; Reis, R. L.; Lima, J. L. F. C.; Fernandes, E. Free Radical Biol. Med. 2006, 40, 632-640. doi:10.1016/j.freeradbiomed.2005.09.017

13. Kalyanaraman, B.; Sohnle, P. G. J. Clin. Invest. 1985, 75, 1618-1622. doi:10.1172/JCl111868
14. Liu, L.; Norman, M. H.; Lee, M.; Xi, N.; Siegmund, A.; Boezio, A. A.; Booker, S.; Choquette, D.; D’Angelo, N. D.; Germain, J.; Yang, K.; Yang, Y.; Zhang, Y.; Bellon, S. F.; Whittington, D. A.; Harmange, J.-P.; Dominguez, C.; Kim, T.-S.; Dussault, I. J. Med. Chem. 2012, 55, 1868-1897. doi:10.1021/jm201331s

15. Guckian, K.; Carter, M. B.; Lin, E. Y.-S.; Choi, M.; Sun, L.; Boriack-Sjodin, P. A.; Chuaqui, C.; Lane, B.; Cheung, K.; Ling, L.; Lee, W.-C. Bioorg. Med. Chem. Lett. 2010, 20, 326-329. doi:10.1016/j.bmcl.2009.10.108

16. Boriack-Sjodin, P. A.; Carter, M. B.; Choi, M. J.; Chuaqui, C.; Deng, Z.; Guckian, K.; Lee, W.; Lin, E. Y.; Sun, L. Substituted Pyrazolones. WO Patent 2007059359 A2, May 24, 2007.

17. Cheng, C.; Shih, Y.-C.; Chen, H.-T.; Chien, T.-C. Tetrahedron 2013, 69, 1387-1396. doi:10.1016/j.tet.2012.11.001

18. Sharma, A.; Vacchani, D.; Van der Eycken, E. Chem.-Eur. J. 2013, 19, 1158-1168. doi:10.1002/chem.201201868

19. Kozhushkov, S. I.; Potukuchi, H. K.; Ackermann, L. Catal. Sci. Technol. 2013, 3, 562-571. doi:10.1039/c2cy20505j

20. Mousseau, J. J.; Charrette, A. B. Acc. Chem. Res. 2013, 46, 412-424. doi:10.1021/ar300185z

21. Neufeldt, S. R.; Sanford, M. S. Acc. Chem. Res. 2012, 45, 936-946. doi:10.1021/ar300014f

22. Engle, K. M.; Mei, T.; Wasa, M.-S.; Yu, J.-Q. Acc. Chem. Res. 2012, 45, 788-802. doi:10.1021/ar200185g

23. Yeung, C. S.; Dong, V. M. Chem. Rev. 2011, 111, 1215-1292. doi:10.1021/cr100280d

24. Sun, C.-L.; Li, B.-J.; Shi, Z.-J. Chem. Rev. 2011, 111, 1293-1314. doi:10.1021/cr100198w

25. Baudoin, O. Chem. Soc. Rev. 2011, 40, 4902-4911. doi:10.1039/c1cs15058h

26. Cho, S. H.; Kim, J. Y.; Kwak, J.; Chang, S. Chem. Soc. Rev. 2011, 40 , 5068-5083. doi:10.1039/c1cs15082k

\section{License and Terms}

This is an Open Access article under the terms of the Creative Commons Attribution License (http://creativecommons.org/licenses/by/2.0), which permits unrestricted use, distribution, and reproduction in any medium, provided the original work is properly cited.

The license is subject to the Beilstein Journal of Organic Chemistry terms and conditions: (http://www.beilstein-journals.org/bjoc)

The definitive version of this article is the electronic one which can be found at: $\underline{\text { doi: } 10.3762 / \text { bjoc. } 9.240}$ 